\title{
Multimodale Bildgebung einer Torpedo- Makulopathie bei einer chinesischen Patientin: ein Fallbericht
}

\author{
Yuhua Ding ${ }^{a}$ Bangtao $\mathrm{Yao}^{\mathrm{b}} \mathrm{Hui} \mathrm{Ye}^{\mathrm{a}} \mathrm{Yan} \mathrm{Yu}^{\mathrm{a}}$ \\ ${ }^{a}$ Department of Ophthalmology, Jiangsu Province Hospital, The First Affiliated Hospital of Nanjing Medical University, Nanjing, China; \\ ${ }^{b}$ Department of Ophthalmology, Lishui District People's Hospital, Nanjing, China
}

\section{Schlüsselwörter}

Torpedo-Makulopathie $\cdot$ multimodale Bildgebung $\cdot$ RPE $\cdot$ OCT-A

\section{Zusammenfassung}

Hintergrund: Als Torpedo-Makulopathie wird eine seltene, angeborene, gutartige und typischerweise torpedoförmige Läsion der temporalen Makula bezeichnet. Ziel der vorliegenden Untersuchung war die ausführliche Beschreibung der Torpedo-Makulopathie einer chinesischen Patientin mittels multimodaler Bildgebung. Fallbericht: Eine 30-jährige Chinesin, bei der sich im Rahmen einer Routineuntersuchung vereinzelte gelblich-weiße Makulaläsionen im rechten Auge gezeigt hatten, stellte sich in unserer Klinik vor. Andere Symptome lagen nicht vor und der bestkorrigierte Visus (best-corrected visual acuity, BCVA) in beiden Augen betrug 6/6. Die funduskopische Untersuchung ergab eine torpedoförmige und leicht hypopigmentierte Läsion in der temporalen Makularegion des rechten Auges. Infrarot-Aufnahmen des Fundus (Infrared fundal, IR) zeigten eine erkennbare Läsionskontur, die horizontal verlaufend und elliptisch konfiguriert war und deren Spitze auf die Fovea centralis der Makula wies. Das mikroperimetrisch untersuchte Gesichtsfeld erschien normal. In der Spectral-Domain optischen
Kohärenztomographie (SD-OCT) zeigte sich eine normale innere Retina mit einer leichten Verdünnung von äußerer Retina und retinalem Pigmentepithel in der temporalen Makularegion sowie einer entsprechend erhöhten choroidalen Reflektivität. Weiterhin fanden sich in der OCT ein Verlust/eine Verdünnung der äußeren Retina mit ausgeprägter Atrophie der intakten ellipsoiden Zone. Die OCT-Angiographie (OCT-A) der choroidalen Kapillarschicht ergab eine erhöhte Dichte der choroidalen Gefäße im Bereich der Läsion, wohingegen die Gefäßversorgung der oberflächlichen und tiefen Schichten normal ausfiel. In der Fundusautofluoreszenz (FAF) zeigte sich ein normales Signal mit einer leichten Hyperautofluoreszenz am nasalen Läsionsrand. Die Fundus-Fluoreszenzangiographie (FFA) der Läsion ergab eine gemischte Fluoreszenz ohne Leckage und morphologische Veränderungen während des gesamten Bildgebungsprozesses.

Schlussfolgerungen: Der vorliegende Bericht enthält erstmals eine umfassende und ausführliche Beschreibung der Torpedo-Makulopathie mittels Fundusphotographie, IR, mikroperimetrischer Untersuchung des Gesichtsfelds, OCT, OCT-A, FAF und FFA. Die multimodale Bildgebung liefert wertvolle und detaillierte Informationen zur weiteren Abklärung der Merkmale und Entwicklung dieser seltenen Erkrankung.

\section{Hintergrund}

Die Torpedo-Makulopathie ist eine seltene, gutartige, klar definierte, angeborene Erkrankung des retinalen Pigmentepithels (RPE), die typischerweise als torpedoförmige Läsion der temporalen Makula erscheint. Sie wurde erstmals 1992 von Roseman und Gass [1] als solitärer, asymptomatischer, hypopigmentierter Nävus des retinalen Pigmentepithels beschrieben. Typischerweise manifestiert sie sich im Fundus in Form einer solitären, ovalen, hypopigmentierten, projektil- oder torpedo-ähnlichen Läsion, die einen sich nach außen erstreckenden keilförmigen Ausläufer aufweist und entlang der horizontalen Raphe verlaufend in Rich-

\section{KARGER}

Fax +497614520714

information@karger.com

www.karger.com (c) 2019 S. Karger GmbH, Freiburg

Accessible online at:

www.karger.com/kop
Hui Ye

Department of Ophthalmology

Jiangsu Province Hospital, The First Affiliated Hospital of Nanjing Medical University

Nanjing, Jiangsu Province, China

oceandeep2006@126.com 

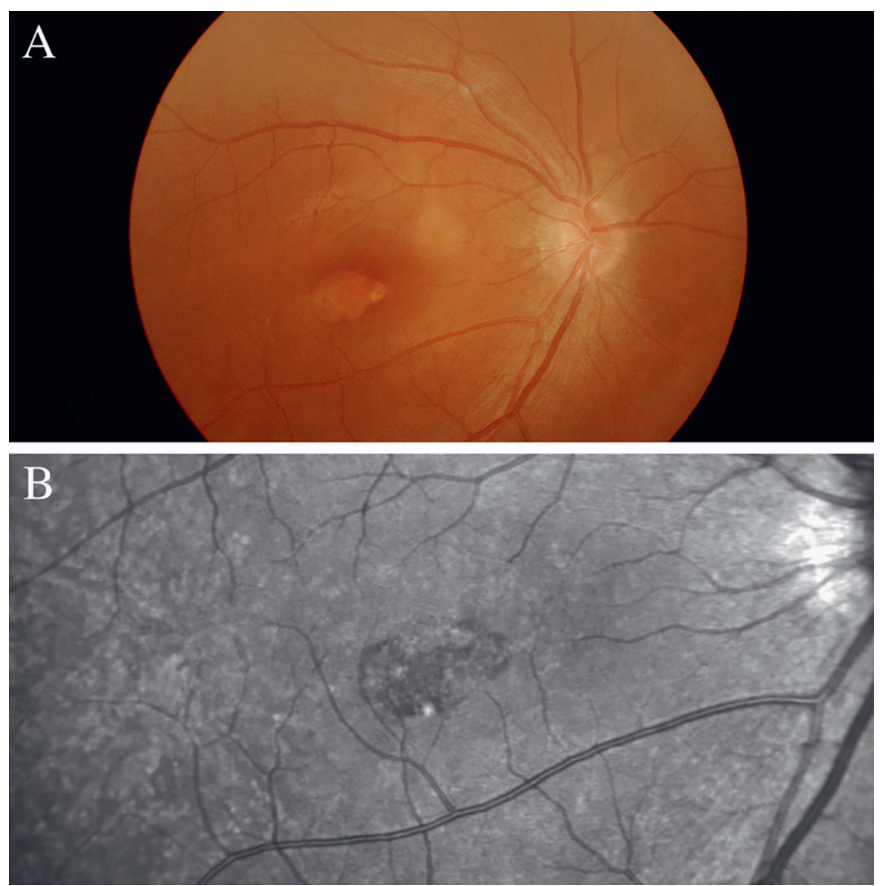

Abb. 1. In der temporalen Makularegion des rechten Auges fand sich eine spindelförmige, gelblich-weiße und hypopigmentierte Läsion von etwa einem halben Discusdurchmesser in der Vertikalachse und einem ganzen Discusdurchmesser in der Horizontalachse, deren Spitze auf die Fovea centralis der Makula wies (a). Die Infrarot-Aufnahme zeigte eine erkennbare Läsionskontur, die horizontal verlaufend und elliptisch konfiguriert war und die der farbigen Fundusphotographie entsprach (b).

tung der Foveola weist [2]. Die Torpedo-Makulopathie erhielt ihren Namen 1993 von Daily [3] wegen ihres typischen Erscheinungsbildes. Der Fundus ist typisch für die Erkrankung und unterscheidet sie von anderen Läsionen, wie Toxoplasma-bedingten Narben, traumatischen Schädigungen, kongenitaler Hypertrophie des RPE (CHRPE) und kongenitaler RPE-Hypertrophie bei Gardner-Syndrom, was die Diagnosestellung ermöglicht.

Die Ätiologie der Torpedo-Makulopathie ist bislang allerdings nicht bekannt. Zahlreiche bildgebende Verfahren sind bei der Untersuchung der Krankheit zum Einsatz gekommen. In der vorliegenden Arbeit verwendeten wir die multimodale Bildgebung, um die Torpedo-Makulopathie einer chinesischen Patientin zu beschreiben. Unseres Wissens ist dies weltweit der erste Bericht über die Untersuchung der Torpedo-Makulopathie mithilfe von Fundusphotographien, Infrarot-Aufnahmen des Fundus, mikroperimetrischer Gesichtsfelduntersuchung, optischer Kohärenztomographie (OCT), OCT-Angiographie (OCT-A), Fundusautofluoreszenz (FAF) und Fundus-Fluoreszenzangiographie (FFA). Wir gehen davon aus, dass es sich in diesem Fall um ein sehr frühes Stadium oder eine leichte Form der Torpedo-Makulopathie handelt.

\section{Fallbericht}

Eine 30-jährige Chinesin, bei der sich im Rahmen einer Routineuntersuchung eine gelblich-weiße Makulaläsion im rechten

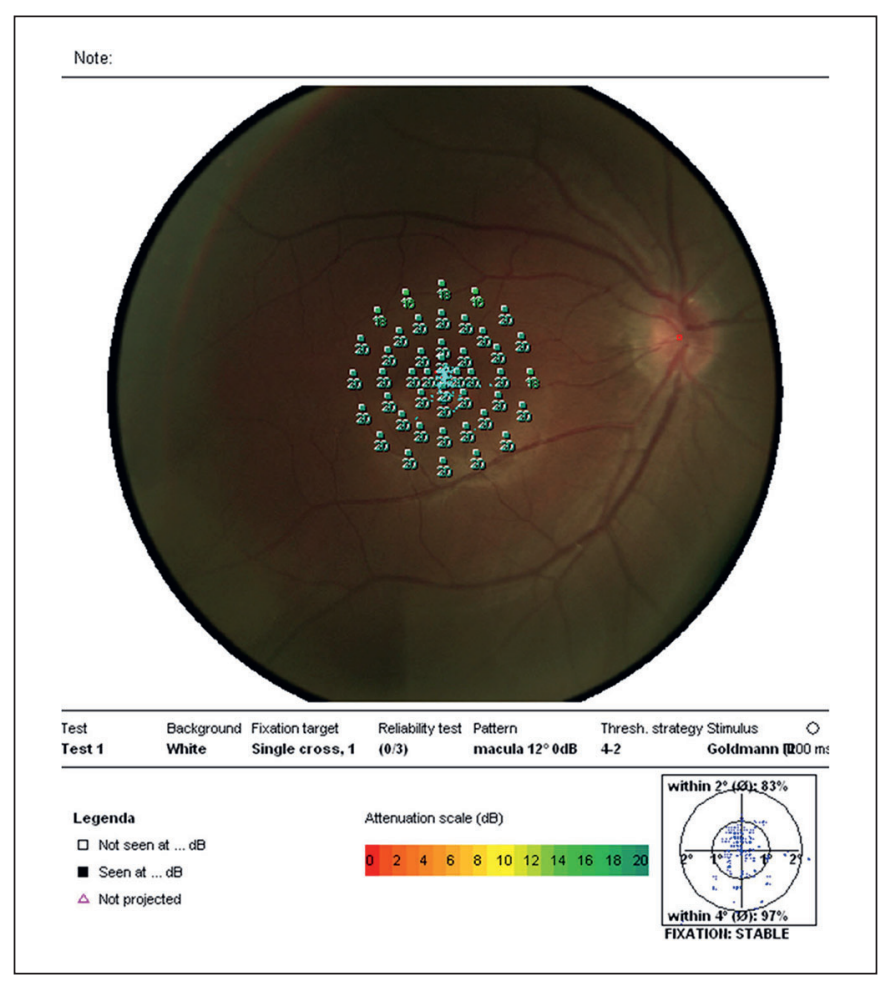

Abb. 2. Die mikroperimetrische Untersuchung des Gesichtsfelds fiel weitgehend normal aus.

Auge gezeigt hatte, stellte sich in unserer Klinik vor. Andere Symptome lagen nicht vor und sie hatte weder Schmerzen noch einen Visusverlust im rechten Auge. Die Patientin hatte in der Vergangenheit kein Trauma erlitten. Ihre medizinische und ophthalmologische Vorgeschichte waren unauffällig. Der initial bestimmte bestkorrigierte Visus lag in beiden Augen bei 6/6. Die Hornhaut war klar und die Untersuchung des vorderen Augensegments war ohne Befund. Die Pupillen waren isokor, rund und zeigten eine Lichtreaktion ohne afferenten Pupillendefekt. Keines der beiden Augen wies eine Katarakt auf. Der initial gemessene Augeninnendruck (intraocular pressure, IOP) betrug 14 $\mathrm{mmHg}$ im rechten Augen und $13 \mathrm{mmHg}$ im linken Auge. Die Funduskopie des linken Auges fiel unauffällig aus. In der temporalen Makularegion des rechten Auges fand sich eine spindelförmige gelblich-weiße und hypopigmentierte Läsion von etwa einem halben Discusdurchmesser in der Vertikalachse und einem ganzen Discusdurchmesser in der Horizontalachse, deren Spitze auf die Fovea centralis der Makula wies (Abb. 1 a). Die Infrarot-Aufnahme zeigte eine erkennbare Läsionskontur, die horizontal verlaufend und elliptisch konfiguriert war und die den farbigen Fundusphotographien entsprach (Abb. 1 b). Die mikroperimetrische Untersuchung des Gesichtsfelds fiel weitgehend normal aus (Abb. 2). In der Spectral-Domain OCT (SDOCT) zeigte sich eine normale innere Retina mit einer leichten Verdünnung von äußerer Retina und retinalem Pigmentepithel in der temporalen Makularegion und einer entsprechend erhöhten choroidalen Reflektivität (Abb. 3). Weiterhin fanden sich in der OCT ein Verlust/eine Verdünnung der äußeren Retina mit 


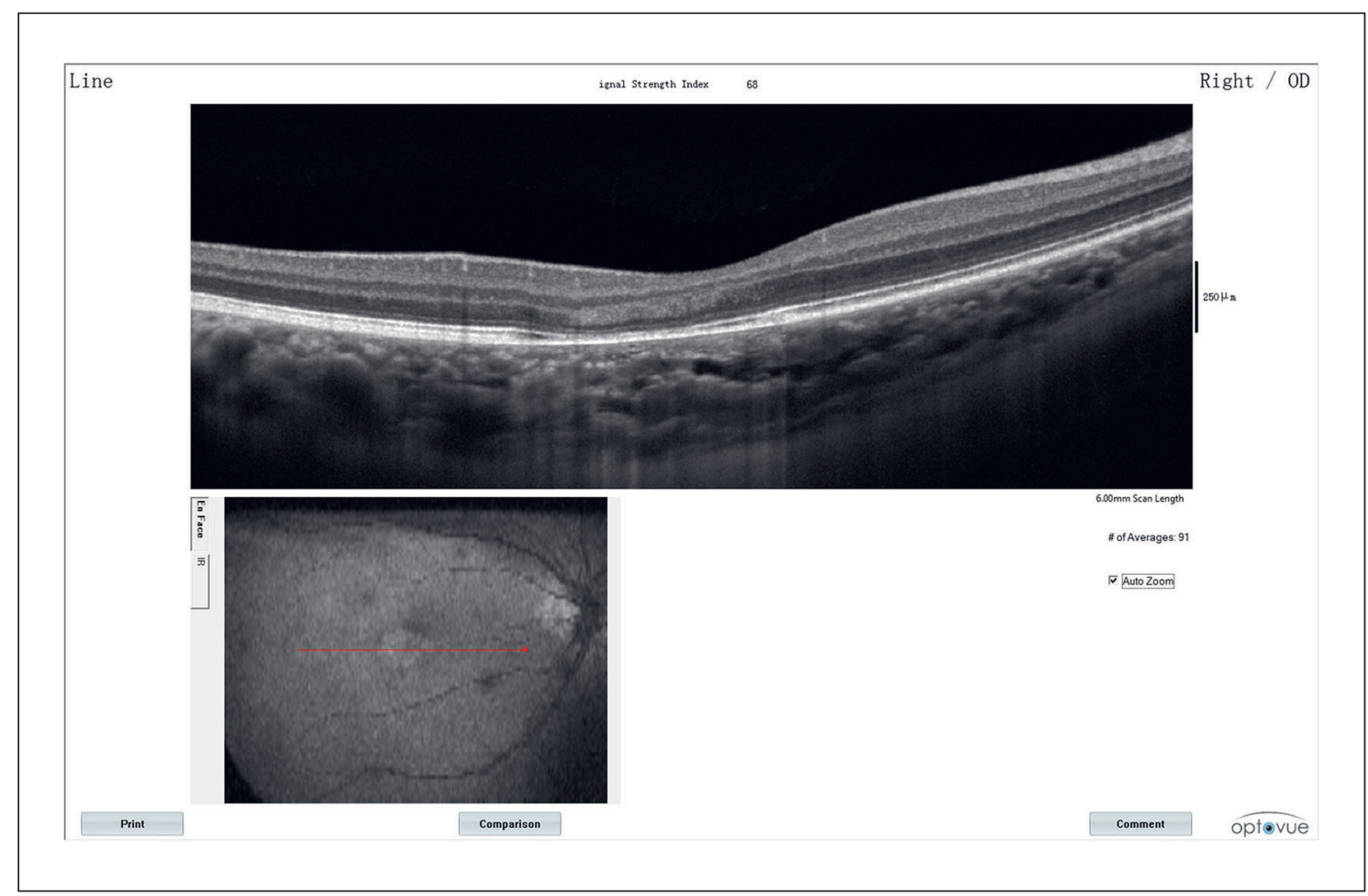

Abb. 3. In der SD-OCT zeigte sich eine normale innere Retina mit einer leichten Verdünnung der äußeren Retina und des retinalen Pigmentepithels in der temporalen Makularegion und einer entsprechend erhöhten choroidalen Reflektivität.

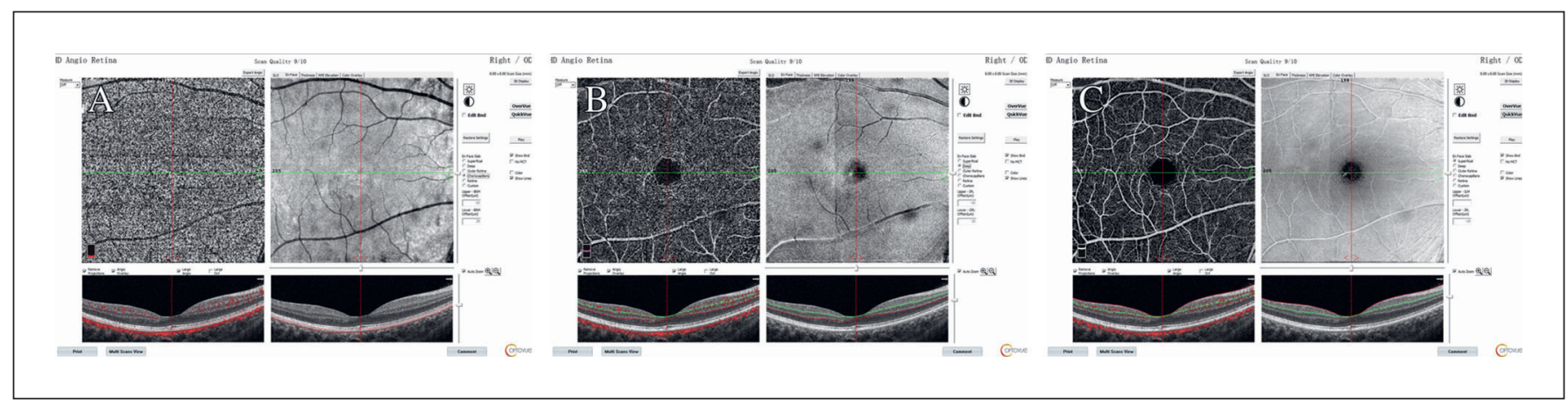

Abb. 4. Die OCT-A der choroidalen Kapillarschicht ergab eine erhöhte Dichte der choroidalen Gefäße im Bereich der Läsion (a). Die OCT-A der tieferen Retinaschichten (b) und der oberflächlichen Retinaschicht (c) fiel normal aus.

ausgeprägter Atrophie der intakten ellipsoiden Zone. Die OCTA der choroidalen Kapillarschicht ergab eine erhöhte Dichte der choroidalen Gefäße im Bereich der Läsion, wohingegen die Gefäßversorgung der oberflächlichen und tiefen Schichten normal ausfiel (Abb. 4). In der Fundusautofluoreszenz (FAF) zeigte die Läsion normale Signale überwiegend mit einer leichten Hyperautofluoreszenz am nasalen Läsionsrand (Abb. 5a). Die FFA der Läsion ergab eine gemischte Fluoreszenz ohne Leckage und morphologische Veränderungen während des gesamten Bildgebungsprozesses (Abb. 5 b-d). Aufgrund dieser Ergebnisse wurde die Diagnose Torpedo-Makulopathie bei dieser Patientin eindeutig bestätigt.

Die Behandlung der Patientin wurde engmaschig überwacht und alle 3 bis 6 Monate erfolgte eine Nachuntersuchung. Ihr bestkorrigierter Visus blieb in beiden Augen bei 6/6 und die Fundusläsion blieb während der 15-monatigen Verlaufskontrolle unverändert.

\section{Diskussion}

Die Torpedo-Makulopathie ist eine seltene, angeborene, gutartige und klar definierte Makulopathie, die gewöhnlich asymptomatisch verläuft, gelegentlich jedoch im Rahmen einer Routineuntersuchung festgestellt wird. Sie tritt häufig bei Patienten ohne relevante medizinische Vorgeschichte und meist unilateral auf; allerdings wurden in der Vergangenheit auch bilaterale Fälle beschrieben [4]. Die Torpedo-Makulopathie erscheint als horizontal verlaufende, ovale, gelblich-weiße hypopigmentierte Läsion in 

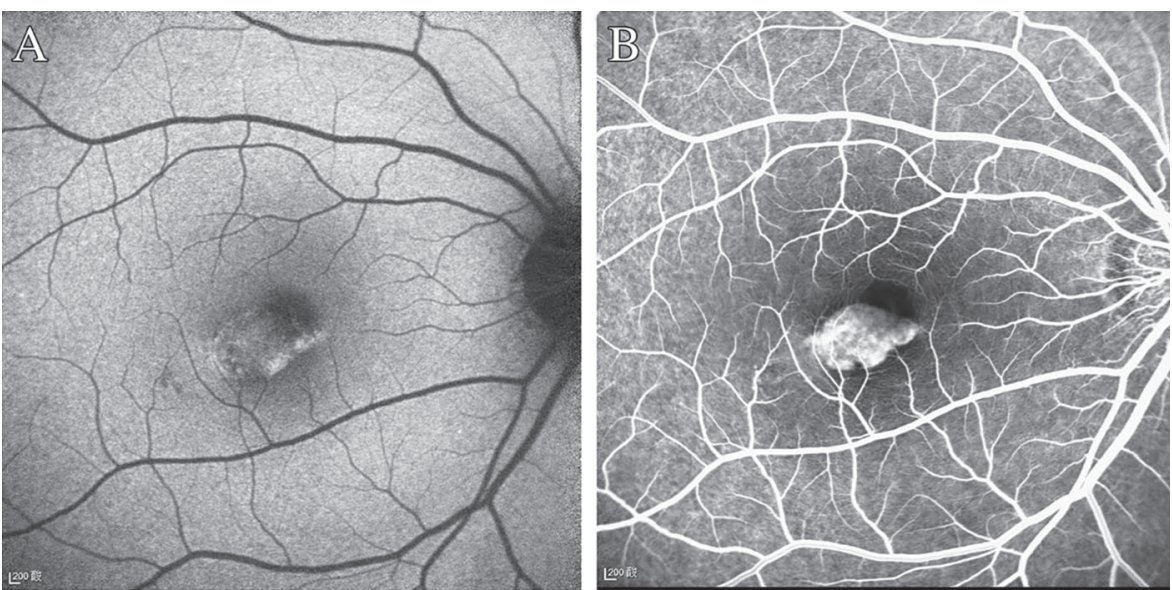

Abb. 5. In der FAF zeigte die Läsion normale Signale überwiegend mit einer leichten Hyperautofluoreszenz am nasalen Läsionsrand (a). Die FFA der Läsion ergab eine gemischte Fluoreszenz ohne Leckage und morphologische Veränderungen während des gesamten Bildgebungsprozesses $(\mathbf{b}-\mathbf{c})$.
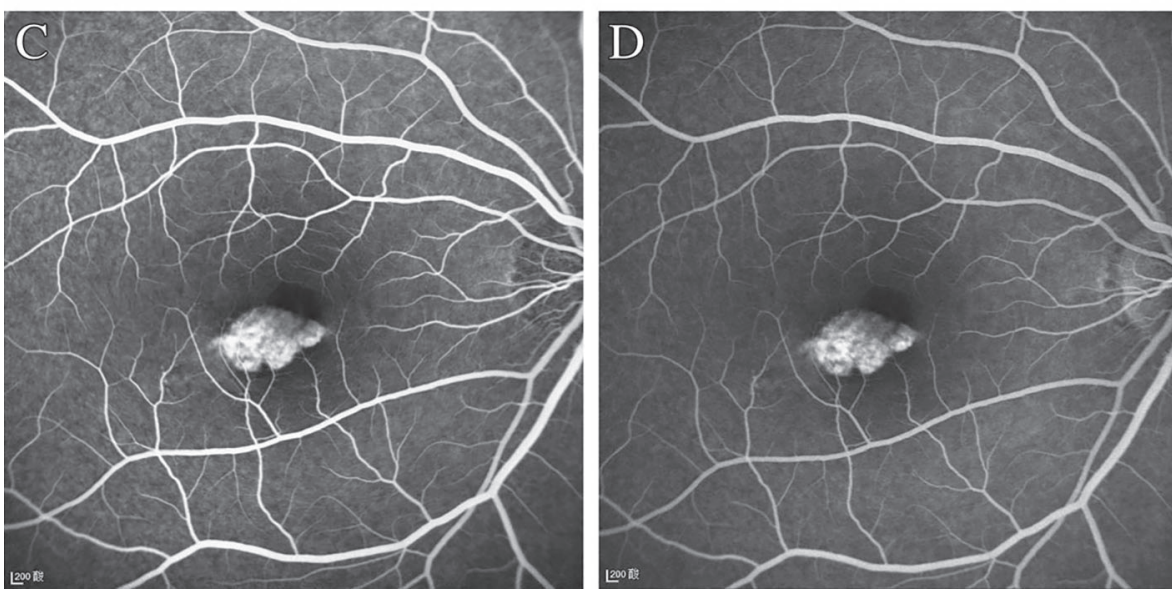

der temporalen Makularegion, deren Spitze auf die Fovea centralis weist.

Die Ätiologie dieser Erkrankung ist bislang nicht bekannt. Es liegen nur wenige Berichte über Studien zur Klärung der Pathogenese dieser Läsion vor. Pian et al. [5] zufolge könnte es sich um eine Entwicklungsstörung innerhalb der Nervenfaserschicht im Bereich der horizontalen Raphe handeln. Shields [6] vermutete, dass die Ursache für die Läsion in einer persistierenden Entwicklungsstörung des retinalen Pigmentepithels während der Ausbildung des embryonale temporale Ausbuchtung liegt. Nach Annahme von Golchet et al. [7] besteht ein möglicher Zusammenhang der Läsion mit einer Fehlbildung des Ausführungskanals von Arteria ciliaris posterior longa und Nerven.

Die mikroperimetrische Untersuchung zeigte präzise die Korrelation von Netzhautsensitivität und Fundusläsion und wies darüber hinaus ein Mikroskotom im zentralen Gesichtsfeld nach, das mit anderen perimetrischen Standardmethoden möglicherweise nicht erkennbar gewesen wäre [8]. Veröffentlichte Berichte haben gezeigt, dass ein Skotom häufig mit einer Torpedo-Läsion assoziiert ist [9-11]. Die Untersuchungen der fokalen RPE-Atrophie ergaben, dass die verminderte Versorgung der inneren Retina mit Metaboliten und Sauerstoff infolge des Verlusts der Lamina choroidocapillaris und der Degeneration der Photorezeptoren mit einer verringerten Netzhautsensitivität assoziiert ist [12]. Der vorliegende Fall unterschied sich von den früheren Berichten, da kein
Mikroskotom zu beobachten war, die Netzhautsensitivität normal erschien und die Patientin über eine gute Sehkraft verfügte. Daher gingen wir davon aus, dass das Ausmaß der RPE-Atrophie in diesem Fall gering war und nicht zu einer Funktionsstörung der darüberliegenden Photorezeptoren geführt hatte.

Die SD-OCT ergab eine Desorganisation der äußeren Retina und zeigte eine signifikante RPE-Atrophie mit einer intakten ellipsoiden Zone. Wir nahmen an, dass das RPE die Funktionsfähigkeit der aufnehmenden Photoreptorenzellen des Außensegments aufrechterhalten hatte, und deswegen die ellipsoide Zone weiterhin intakt war und die Patientin ein normales Sehvermögen aufwies. Evan et al. [13] identifizierten 2 Muster von Anomalien: Typ 1: Verdünnung der äußeren Retinastrukturen ohne Kavitation der äußeren Retina und Typ 2: Fälle, in denen sowohl eine Verdünnung der äußeren Retinastrukturen als auch eine Kavitation der äußeren Retina vorliegt. Nach ihrer Theorie gehörte diese Patientin zur Typ-1-Torpedo-Makulopathie. Ferner beobachteten sie, dass Patienten mit Typ-1-Läsion (Alter: 4-37 Jahre) tendenziell jünger waren als solche mit Typ-2-Läsion (Alter: 13-73 Jahre) [13]. Unsere Patientin war 30 Jahre alt und entsprach damit den von ihnen angegebenen Charakteristika.

Die OCT-A erlaubt es, nicht invasiv die Erythrozytenbewegung $\mathrm{zu}$ bestimmen sowie das retinale und choroidale Gefäßsystem darzustellen. Daher können Veränderungen der Lamina choroidocapillaris mittels OCT-A sichtbar gemacht werden. Papastefanou 
und Kollegen [14] haben die Charakteristika von Torpedo-Läsionen mithilfe der OCT-A beschrieben und die choroidale Gefäßsegmentierung mit Hyporeflektivität (Atrophie) nachgewiesen, die wiederum mit der OCT des subretinalen Spalts korreliert war. Bei unserer Patientin war jedoch kein subretinaler Spalt zu beobachten, und die OCT-A-Ergebnisse fielen abweichend aus. Die OCT-A des choroidalen Kapillarsegments ergab eine erhöhte choroidale Gefäßdichte und zeigte eine Verdünnung des RPE als optische Signalweiterleitung bei erhöhter Dicke der Choroidea; die oberflächlichen und tiefen Schichten erschienen dagegen normal. Der Vergleich unseres Falles mit früher berichteten anderen Fällen [14] ergab, dass es sich in unserem Fall um ein Frühstadium gemäß OCT-Klassifikation handelte.

Das Autofluoreszenzsignal ist vorwiegend durch das Lipofuszin im RPE bedingt [15]. In unserem Fall zeigte die FAF normale Signale überwiegend mit einer leichten Hyperautofluoreszenz im nasalen Bereich der Läsion. Eine mögliche Erklärung hierfür ist in der Verdünnung des RPE und der äußeren nukleären Schicht zu sehen.

Die FFA der Läsion ergab eine gemischte Fluoreszenz ohne Leckage und morphologische Veränderungen während des gesamten Bildgebungsprozesses. In diesem Fall war keine choroidale Neovaskularisation $(\mathrm{CNV})$ zu beobachten, wohingegen in einigen wenigen anderen Fällen das Vorliegen einer CNV nachweisbar war [16]. In unserem Fall waren die Läsionen noch auf das RPE begrenzt und eine CNV bestand nicht.

Differentialdiagnosen wie die posteriore Uveitis sollten sorgfältig ausgeschlossen werden. Die posteriore Uveitis, die auch als Choroiditis bezeichnet wird, ist nicht eindeutig definiert. Sie ist durch eine Glaskörperexsudation und choroidale Vasodilatation gekennzeichnet, die zur einer CNV und Sehkraftminderung führen. Die Untersuchungsergebnisse der OCT-A, FFA und FAF bei posteriorer Uveitis unterscheiden sich von denen der TorpedoMakulopathie. In aktiven Choroiditis-Läsionen war in der FFA eine in der Frühphase auftretende Hypofluoreszenz gefolgt von einer Leckage in der Spätphase zu beobachten. Dagegen zeigten die geheilten Läsionen eine Hypofluoreszenz in der Frühphase bei Färbung, jedoch keine Leckage in der Spätphase. Bei aktiven Choroiditis-Läsionen war in der FAF eine nicht eindeutig abgrenzbare Hyperautofluoreszenz zu beobachten, wohingegen sich bei geheilter Choroiditis abgerundete Ränder und eine Hypoautofluoreszenz der Läsionen fanden [17]. Die Befunde unterscheiden sich deutlich von denen einer Torpedo-Makulopathie.

Die vorliegende Studie weist jedoch etliche Limitationen auf. Die Stichprobengröße der Studie war gering und es erfolgte keine Indocyaningrün-Angiographie, die bei der Abgrenzung der Differentialdiagnosen dieser Erkrankung hilfreich ist. Eine weitere Limitation dieser Studie ist darin zu sehen, dass keine weitere Untersuchung zur Verlaufskontrolle vorgesehen ist.

\section{Schlussfolgerungen}

Erstmals wurde ein Fall von Torpedo-Makulopathie vorgestellt, bei dem Fundusphotographien, Infrarot-Aufnahmen des Fundus, eine mikroperimetrische Untersuchung des Gesichtsfelds, OCT, OCT-A, FAF und FFA eingesetzt wurden. Diese ergaben, dass es sich um ein Frühstadium oder einem leichten Typ von TorpedoMakulopathie handeln könnte. Der natürliche Verlauf der Torpedo-Makulopathie ist weiterhin unklar, und es ist nicht bekannt, ob sie sich sehr langsam oder gar nicht weiterentwickelt. Die multimodale Bildgebung liefert wertvolle und detaillierte Informationen, welche die Diagnose und das Verständnis dieser seltenen Erkrankung erleichtern. Es handelt sich um eine sehr seltene Krankheit, und weltweit sind weitere Fallberichte und eine längere Verlaufskontrolle erforderlich, um die Ätiologie, Merkmale und Entwicklung der Läsion besser zu verstehen.

\section{Danksagung}

Nicht zutreffend.

\section{Autorenbeiträge}

Y. H. D. erhob die Daten der Patientin, durchsuchte die Literatur und erstellte das Manuskript; B. T. Y. nahm die redaktionelle Bearbeitung des Manuskripts vor und erstellte die Abbildungen. H. Y. lieferte hilfreiche Vorschläge zur Erstellung des Manuskripts und verbesserte es so. Y. Y. stellte als behandelnder Arzt im vorliegenden Fall die Diagnose und gab die eingereichte Version zur Veröffentlichung frei. Alle Autoren haben die finale Fassung des Manuskripts gelesen und freigegeben.

\section{Verfügbarkeit der Daten und Materialien}

Die in dieser Arbeit verwendeten und/oder analysierten Datensätze sind auf begründete Anfrage vom Korrespondenzautor erhältlich.

\section{Zustimmung zur Veröffentlichung}

Die schriftliche Einwilligungserklärung der Patientin lag vor.

\section{Interessenkonflikte}

Die Autoren erklären, dass keine Interessenkonflikte bestehen.

\section{Lizenzangabe}

Yuhua Ding, Bangtao Yao, Hui Ye, Yan Yu: Multimodal imaging of torpedo maculopathy in a Chinese woman: a case report. BMC Ophthalmology 2019; 19:54 (https://doi.org/10.1186/s12886-019-1161-7), (c) The Author(s), 2019 (Übersetzung, "Abbrevations», «Acknowledgements», «Funding», «Ethic's approval and consent to publish» und «Publisher's note» gekürzt), lizensiert unter CC BY 4.0 (https://creativecommons.org/licenses/by/4.0/deed.de). 


\section{References}

1 Roseman RL, Gass JD: Solitary hypopigmented nevus of the retinal pigment epithelium in the macula. Arch Ophthalmol 1992;110: 1358-1359.

2 Trevino R, Kiani S, Raveendranathan P: The expanding clinical spectrum of torpedo maculopathy. Optom Vis Sci 2014;91:S71-S78.

3 Golchet PR, Jampol LM, Mathura JR, et al. Torpedo maculopathy. Br J Ophthalmol 2010; 94:302-306.

4 Richez F, Gueudry J, Brasseur G, et al.: Bilateral torpedo maculopathy. J Fr Ophtalmol 2010; 33:296.

5 Pian D, Ferrucci S, Anderson SF, et al. Paramacular coloboma. Optom Vis Sci 2003; 80:556-563.

6 Shields CL, Guzman JM, Shapiro MJ, et al. Torpedo maculopathy at the site of the fetal "bulge" Arch Ophthalmol. 2010;128:499-501.
7 Golchet PR, Jampol LM, Mathura JR, Jr, et al.: Torpedo maculopathy. Br J Ophthalmol 2010; 94:302-306

$>8$ Sunness JS, Schuchard RA, Shen N, et al.: Landmark-driven fundus perimetry using the scanning laser ophthalmoscope. Invest Ophthalmol Vis Sci 1995;36:1863-1874.

$>9$ Su Y, Gurwood AS: Neurosensory retinal detachment secondary to torpedo maculopathy. Optometry 2010;81:405-407.

10 Sanabria MR, Coco RM, Sanchidrian M: OCT findings in torpedo maculopathy. Retin Cases Brief Rep 2008;2:109-111.

1 Barbazetto IA, Maris PJ, Jr, Greenstein VC: Solitary albinotic spot of the retinal pigment epithelium: a functional and imaging study. Klin Monatsbl Augenheilkd 2008;225: 295-297.

12 Midena E, Vujosevic S, Convento E, et al.: Microperimetry and fundus autofluo- rescence in patients with early age-related macular degeneration. Br J Ophthalmol. 2007;91:1499-1503.

13 Wong EN, Fraser-Bell S, Hunyor AP, et al.: Novel optical coherence tomography classification of torpedo maculopathy. Clin Exp Ophthalmol 2015;43:342-348.
14 Papastefanou VP, Vázquez-Alfageme C, Keane PA, et al.: Multimodal imaging of torpedo maculopathy with swept-source, en face optical coherence tomography and optical coherence tomography angiography. Retin Cases Brief Rep 2018;12:153-157.

15 von Rückmann A, Fitzke FW, Bird AC: Distri bution of fundus autofluorescence with a scanning laser ophthalmoscope. Br J Ophthalmol 1995;79:407-412.

6 Jurjevic D, Böni C, Barthelmes D, et al.: Torpedo maculopathy associated with choroidal neovascularization. Klin Monatsbl Augenheilkd 2017;234:508-514.

17 Aggarwal K, Agarwal A, Sharma A, et al.: Detection of type 1 choroidal neovascular membranes using optical coherence tomography angiography in tubercular posterior uveitis. Retina 2018;23. 